\title{
Resource scarcity and outcome conflict in time-sharing performance
}

\author{
PAMELA S. TSANG and TONYA L. SHANER \\ Wright State University, Dayton, Ohio \\ and \\ MICHAEL A. VIDULICH \\ Armstrong Laboratory, Wright-Patterson Air Force Base, Ohio
}

\begin{abstract}
The efficacy of the resource-scarcity and outcome-conflict views in explaining dual-task interference was examined. A discrete-continuous task pair was purposely chosen to allow fine-grained analysis of time-shared performance. The relative priority of the dual task was manipulated by a secondary task technique to test for performance tradeoff that would be indicative of resource allocation. The temporal predictability of the discrete stimuli was manipulated to examine possible strategic avoidance of interference. The moment-by-moment data did not reveal any evidence for a switching strategy. It was concluded that the intricate interference patterns could be more easily interpreted within the resource framework than within the outcome-conflict framework.
\end{abstract}

Time-shared performance typically degrades from single-task performance level. The present study examines the viability of two explanations for this degradation: resource scarcity and outcome conflict.

First, it is important to make clear what is meant by timesharing performance. Time-sharing in the computer domain refers to several terminals accessing a central processor at different times. In other words, one central processor serves several terminals by rapidly switching between them. Time-sharing in this sense is sometimes used in the psychological literature. For example, Hirst and Kalmar (1987) used time-sharing to refer to rapid switching between the processing of two tasks. In contrast, time-sharing in the present paper is used along the tradition followed by Gopher (1993), Friedman, Polson, and Dafoe (1988), Moray (1967), and Wickens (1990). Here, time-sharing refers to the simultaneous processing of two or more tasks.

\section{Resource Theory}

According to resource theories (e.g., Gopher, 1986; Kahneman, 1973), time-shared performance will compete for limited resources if certain conditions are met.

Support was provided in part by National Institute of Aging Grant AG08589, and in part by Southeastern Center for Electrical Engineering Education, Task SCEEE-HER/89-0005, subcontract under Air Force Aerospace Medical Research Laboratory, Contract No. F3361588-D-0532 (Gary B. Reid was the technical monitor). The authors thank Rick Backs for his comments at various stages of the project, and Scott Braum and Kevin Lew for their assistance in data collection. The authors also thank Art Kramer, two anonymous reviewers, and Stu Klapp for their careful reading and thoughtful comments on an earlier version of the manuscript. Correspondence should be addressed to P. S. Tsang, Department of Psychology, Wright State University, Dayton, $\mathrm{OH} 45435$ (e-mail: ptsang@desire.wright.edu).
The necessary conditions are that the time-shared tasks are performed simultaneously, they are resource-limited, and their combined resource demand exceeds resource availability. Resource competition is manifested in performance decrements from the single-task level. However, performance of the time-shared tasks need not degrade to the same extent. This is because a central tenet of resource theories is that various amounts of resources can be flexibly allocated to the simultaneous activities. For example, Kahneman (1973) proposed that time-shared performance is continuously monitored and evaluated so that resource allocation between tasks can be continuously modulated via a closed feedback loop.

Empirically, resource allocation can be induced by manipulating the relative priority of the time-shared tasks with the secondary task technique (see O'Donnell \& Eggemeier, 1986; Ogden, Levine, \& Eisner, 1979; Rolfe, 1971). The assumption is that as the priority of one task increases, additional resources will be allocated to that task in order to maintain a certain level of performance. With limited resources, allocating more resources to one task necessarily leaves less for the other. The primary task is thus protected at a cost to the concurrent secondary task performance. This is referred to as performance tradeoff and is interpreted as an indication of resource allocation (Kinchla, 1980; Navon, 1985a; Sperling \& Melchner, 1978). Resource theories posit that differential decrements in the time-shared tasks can be brought about by resource allocation that is subject to voluntary control

Several mechanisms have been proposed to account for the resource effects (e.g., limited attentional capacity proposed by Kahneman, 1973, competition for brain space proposed by Kinsbourne \& Hicks, 1978). All of the proposed mechanisms share the idea that scarcity of 
something is the cause of time-sharing decrements (Wickens, 1986b). Studies supporting the resource explanation abound in the literature (e.g., Beatty, 1982; Fracker \& Wickens, 1989; Friedman et al., 1988; Gopher, Brickner, \& Navon, 1982; Tsang \& Wickens, 1988) and will not be reviewed here.

\section{Outcome Conflict}

Recently, there have been concerns as to whether the notion of resource is necessary to explain performance degradation in time-shared tasks. One cause for these concerns is that there are conceivable nonresource mechanisms that could explain dual-task decrements equally well. One of several potential mechanisms is the outcome conflict proposed by Navon (1984). Navon (1985b) refers outcome conflicts to any "outputs, throughput, or side-effects that are harmful to the processing of the concurrent task" (p. 7). Whereas the resource view posits the scarcity of something as the cause of dual-task interference, the outcome conflict suggests that the interference is a result of an overabundance of processing (Wickens, 1986b).

\section{The Present Study}

The present research was designed to examine the simultaneous performance of a discrete task and a continuous task. The combination of a discrete and a continuous task was chosen for two reasons. First, it is difficult to ascertain the simultaneity of processing of two discrete tasks even if they were presented simultaneously. Simultaneous processing is, of course, a necessary condition for interference to occur according to both the resource and the outcome-conflict explanation. Second, it is difficult to ascertain the nature of the interference (such as the onset, the duration, and the magnitude of the interference) between two continuous tasks. A discretecontinuous task pair provides a unique condition in which the precise moment that the discrete stimulus occurred is known, and any intrusive effect from processing it should be evident in the concurrent continuous performance.

A continuous tracking task ${ }^{1}$ and a discrete Sternberg memory task were used in the present study. The tracking task had a continuous input signal and was responded to with an analog joystick. Because of the continuous input, the tracking task required continuous processing of the input signal and the response. Two tracking measures were examined: (1) tracking error was the distance between the cursor and the target, and (2) tracking control speed was the amount the joystick had moved per unit time. Tracking error was a precision measure, whereas control speed measured how much the subject moved the stick. Control speed was a more direct measure of the motor response than was tracking error. Moment-bymoment tracking performance allowed a fine-grained analysis of the time-course of intrusion of one task upon the performance of another.

The memory task had a discrete stimulus onset and offset and was responded to with a discrete buttonpress.
However, the memory task also had a continuous component-namely, maintaining the memory set in working memory throughout the trial. Thus, there should have been some concurrent demand from the two tasks throughout the trial.

Assuming that the tracking and memory tasks competed for some common resources, processes, or mechanisms, both the resource and the outcome-conflict explanation would predict dual-task decrement. The present study therefore also manipulated the relative priority of the time-shared tasks to induce performance tradeoff. While performance tradeoff is an inherent product of resource scarcity, it would be difficult to attribute priorityinduced performance tradeoff to an abundance of something or outcome conflict.

To induce performance tradeoff, the secondary task technique was used. Contrary to convention, priority was a between-subject manipulation in the present studythe tracking and memory tasks were designated as primary task for separate groups of subjects. This was to heed Navon's (1984) concern that providing differential performance expectations for both tasks may present demand characteristics that lead subjects to believe that the two task performances should tradeoff. Navon (1984) has argued that the two common priority manipulation procedures may have major methodological problems. One procedure explicitly asks subjects to divide their attention in various proportions between the tasks. For example, subjects might be instructed to allot $75 \%$ of their resources to one task and $25 \%$ to the other (e.g., Gopher et al., 1982; Sperling \& Melchner, 1978). The other procedure varies the ratio of the levels of minimal performance requirements for the two tasks (e.g., Gopher \& Navon, 1980; Wickens \& Gopher, 1977). A high level of performance for the primary task is required, and a performance standard is provided for the subjects. At the same time, a lower performance standard is provided for the secondary task. In some cases, an on-line performance indicator was used to encourage the subjects to conform to the standards. According to Navon (1984), these two procedures have a built-in bias against maximization of joint performance, because they suggest to the subjects that the task performances are expected to tradeoff. Performance tradeoffs observed under these procedures may mean nothing more than the subjects' complying with demand characteristics.

The present study minimized the influence of these demand characteristics by using a between-subject design. Each subject always performed the same task as the primary task. This was expected to provide no opportunity for the subjects to shape their primary and secondary task performance to conform to "performance expectation." Also, the priority instructions used in the present study were carefully designed to neither suggest nor encourage degradation of the secondary task.

Another major manipulation in the present study was the manipulation of the temporal predictability of the presentation of the memory probes. Perfectly predictable stimulus arrival should be more conducive to the devel- 
opment of strategy that could better protect the primary task performance (Broadbent, 1987; Moray, 1978; Welford, 1978). For example, resources could be modulated in a more timely and precise fashion, leading to better protection of the primary task. In theory, the primary task performance could also be protected by avoiding outcome conflict. To avoid outcome conflict, simultaneity of common stages of processing, common processes, or common mechanisms must be avoided. This could be accomplished by switching. Switching refers to putting one task on hold while performing the other and alternating between tasks rapidly. One main purpose of the momentby-moment tracking error analysis was to check for these possibilities. Dual-task studies employing at least one tracking task will be briefly reviewed below.

\section{Dual-Task Interference}

Cliff (1973) had subjects time-share a continuous tracking task with a verbal shadowing task. Holds for brief periods of time were found in both the tracking and the shadowing performance. Onstott (1976) found control shifting between two axes of a continuous tracking task. McLeod (1977) had subjects time-share a visual-manual discrete tracking task with a discrete auditory tone identification task that was responded to manually or by speech. A lower probability of tracking response was observed $300-400 \mathrm{msec}$ before the manual response to the tone, but not before the speech response. Fracker and Wickens (1989) also found that their subjects occasionally employed a switching strategy, but they found their subjects to spend most of their time controlling two visual-manual continuous tracking tasks simultaneously.

The above studies had subjects time-share either two continuous tasks or two discrete tasks. Five other studies are especially relevant to the present one in that a discrete-continuous task pair was used and relative priority between the time-shared tasks was manipulated. Klapp, Kelly, and Netick (1987) had subjects perform a visual-manual pursuit tracking task and an auditorymanual reaction time task. More tracking holds (hesitations) were observed when the tracking task was performed with the reaction time task than when it was performed without. Tracking holds were observed only when an explicit concurrent discrete response was required in a second study (Netick \& Klapp, 1994). Vidulich (1990) had subjects time-share a visual-manual unstable tracking task with a visual-manual Sternberg memory task. A temporary tracking-error increase in response to the memory probe was observed, and the error increased with increased memory response complexity. In another study, Vidulich (1989) had subjects time-share a visualmanual compensatory tracking task with the same Sternberg memory task. Transient tracking-error increase and transient decrease in control speed around the occurrence of the memory probe were observed. Lastly, Tsang and Wickens (1988) had subjects time-share a visualmanual compensatory tracking task with another tracking task or with a running memory task that could be presented visually or auditorily and responded to manu- ally or by speech. The difficulty of the tracking task varied continuously between first- and second-order control within a trial. Error spikes in the tracking performance were observed in response to the tracking-difficulty increase when the tracking task was paired with the memory task but not with another tracking task. These studies collectively show that increased tracking error, reduced control speed, and tracking holds associated with the concurrent discrete response were common forms of interference.

A variety of tasks were time-shared with the tracking task in the studies reviewed. Yet, they all reported clear dual-task interference, provided that the tasks were sufficiently difficult (Cliff, 1973; Tsang \& Wickens, 1988; Vidulich, 1990). Interference was observed between two visual tasks (Fracker \& Wickens, 1989; Onstott, 1976; Tsang \& Wickens, 1988; Vidulich, 1989, 1990), between one visual and one auditory task (Cliff, 1973; Klapp et al., 1987; McLeod, 1977; Netick \& Klapp, 1994; Tsang \& Wickens, 1988), between two tracking axes controlled by the same hand (Onstott, 1976), between two tasks controlled by two hands (Fracker \& Wickens, 1989; Klapp et al., 1987; McLeod, 1977; Netick \& Klapp, 1994; Tsang \& Wickens, 1988; Vidulich, 1989, 1990), and between two tasks, one controlled manually and one by speech (Cliff, 1973; Tsang \& Wickens, 1988). It would be difficult to attribute all of these interferences to peripheral interference due to performing two tasks of the same input modality, of the same output modality, or with the same physical apparatus. The present research will more deeply explore the nature of interference with primarily cognitive or central processing manipulations.

\section{Research Objectives}

The primary objective of the present study was to distinguish between the resource-scarcity and outcomeconflict explanations of dual-task interference. A discretecontinuous task pair was used. The two main manipulations were the relative priority between the time-shared tasks and the temporal predictability of the arrival of the discrete stimulus. Priority was manipulated to test for graded-performance tradeoff that would be indicative of resource allocation. Temporal predictability was manipulated to examine possible strategic avoidance of interference. Moment-by-moment performance was examined to allow fine-grained analysis of the interference. One important distinction in the manifestation of the resource-scarcity and outcome-conflict effects is that resource theories allow graded-performance changes in response to the priority or predictability manipulations. In contrast, outcome conflict can only be avoided by avoiding simultaneity of the competing components. This could be accomplished by switching between tasks.

\section{METHOD}

\section{Subjects}

Fifteen male and 15 female right-handed college students, between the ages of 18 and 53 years $(M=24)$, received research credit 
or hourly pay for their participation. All subjects reported normal or corrected-to-normal vision and normal color vision.

\section{Tasks}

A discrete Sternberg memory task (Sternberg, 1969) and a continuous tracking task were used. Each task had two levels of difficulty to test that the task was resource-limited. Each trial was $3 \mathrm{~min}$ in duration.

Sternberg task (SB). At the beginning of each Sternberg task, a memory set of either two (SB2) or four (SB4) randomly selected English consonants were presented. The memory probes were presented one at a time at the vertical center of the screen throughout the trial. The probe subtended a vertical visual angle of $17^{\prime}$ ' and a horizontal visual angle of 13'. The subjects pressed the "yes" button if the probe belonged to the memory set and the "no" button otherwise with the left hand. Each probe remained on the screen until the subject had responded. The subjects were inst ructed to respond as rapidly as possible while maintaining 100\% accuracy.

The first memory probe appeared $5 \mathrm{sec}$ into the trial. Temporal predictability of the memory probes was manipulated by using three paces of presentation: fixed interstimuli interval (Fixl), variable interstimuli interval (VarI), and fixed response-stimulus interval (FixR). The interstimuli interval (ISI) for FixI was fixed at $5 \mathrm{sec}$, with 35 probes presented in each trial. For the VarI condition, an average ISI of $5 \mathrm{sec}$ with a variability window of $1 \mathrm{sec}$ (i.e., $5 \sec \pm 1$ ) was used. That is, the next stimulus was presented between 4 and $6 \mathrm{sec}$ after the last stimulus. The total number of probes per trial with the VarI condition ranged from 33 to 36 . For the FixR condition, a fixed response-stimulus interval (RSI) of $4.4 \mathrm{sec}$ was used in the first session. The RSI value was chosen such that the total number of probes displayed would approximate that displayed in the FixI condition. In Session 2, an individualized RSI value was obtained by subtracting the subject's Session 1 mean reaction time (RT) from $5 \mathrm{sec}$. In Sessions 3 and 4, the RSI was adjusted again using the subject's mean RT from Session 2. The total number of probes per trial for the FixR condition ranged from 31 to 36 . The subjects were randomly assigned to one temporal predictability condition.

Both the FixI and the FixR conditions were considered to have predictable stimulus arrival. However, it is possible that a response executed by the subject would serve as a better time-marker than would an externally controlled fixed-pace stimulus. The selfgenerated time-marker could perhaps be used more effectively to anticipate the next memory probe, enabling the FixR group to better monitor the level of attention required to protect the tracking task or to monitor for an opportune time to switch between tasks.

Recorded for each trial were percent correct, percent omitted, and median RT for the correct responses. In addition, the onset of each memory probe was time-stamped throughout the trial.

Tracking task (TR). The tracking task was a continuous onedimensional compensatory tracking task, performed by the right hand. The subjects were to maintain a laterally moving cursor on a stationary reference line located at the center of the screen. A unique sum of five sines was used to generate the random disturbance for each trial. The bandwidth for the five sines were $0.13044,0.22222,0.37500,0.63830$, and $1.11111 \mathrm{~Hz}$. The tracking cursor was a vertical line that subtended a visual angle of $26^{\prime}$. A Measurement System Inc. Model 531 spring-centered, fingercontrolled joystick was used. First-order, or velocity, control (TR1) and second-order, or acceleration, control (TR2) were used.

The direction of the joystick deflection determined the direction of travel. For first-order control, the amount of deflection directly determined the speed of the cursor; the cursor had zero velocity when the stick was centered and maximum velocity at maximum stick deflection. For second-order control, the amount of deflection determined the acceleration of the cursor. Zero deflection here meant zero acceleration but not necessarily zero velocitythe cursor would keep moving at a constant speed when the stick was centered. To change direction, the accelerating cursor first had to decelerate in the direction in which it was currently headed before it could start accelerating again in the opposite direction. Therefore, direction reversal of the cursor was not instantaneous as the direction of the stick deflection was changed. Anticipation of where the cursor might end up as a result of the stick input was of utmost importance for good control of an acceleration system.

The joystick position was sampled 30 times per second. Ten samples constituted a frame $(333 \mathrm{msec})$. There were 3 frames per second and 540 frames per trial $(180 \mathrm{sec})$. For each frame, a mean stick position and a root mean square error (RMSE) were calculated. RMSEs were scaled such that 0 represented no error (when the cursor was at the reference line) and 1 represented maximum error (when the cursor was at the farthest position from the center on either side). A running average of the RMSEs was obtained over a l-sec sliding window. Finally, a mean RMSE of these running averages was calculated for the trial. Tracking control speed was obtained by taking the absolute value of the difference between the mean stick position of the previous frame and the current frame. Since the time for each frame was constant, the change in stick position per frame represented the average speed of movement at that point in time. A running average of the control speed was obtained over a 1 -sec sliding window. A mean of these running averages of control speed was calculated for the trial. In sum, we have a RMSE and control speed for each frame (used in the moment-by-moment analysis) and a trial mean based on the running averages for RMSE and control speed for each trial.

Dual tasks. A pairwise combination of two single tasks, each with two levels of difficulty, yielded four dual-task conditions: TR1SB2, TR1SB4, TR2SB2, and TR2SB4. To minimize visual scanning between the two tasks, the tracking task was displayed closely below the memory task. The vertical visual angle spanning the top of the memory probe and the bottom of the tracking target was $57^{\prime}$. The farthest cursor position from center and the memory probe subtended a visual angle of $3^{\circ} 17^{\prime}$.

The memory task and the tracking task had in common their input and output modalities. Both were visually presented and responded to manually. Within Wickens's (1990) multiple-resource framework, the memory task was primarily a verbal task that placed the heaviest demand on the central processing resources. Response requirements for this task were minimal. The tracking task was primarily a spatial task that placed the heaviest demand on the responseprocessing resources. Second-order, or acceleration, control required anticipation of the future position of the stimulus and generation of leads for effective control, placing additional demand on the central processing and response-processing resources (e.g., Wickens, Gill, Kramer, Ross, \& Donchin, 1981). Resource competition between the memory and second-order tracking tasks would therefore be heightened, relative to first-order control dual tasks.

On-line feedback On-line feedback was intended to augment the subjects' awareness of their moment-by-moment performance. The on-line feedback was integrated with the experimental tasks so that the subjects would not have to look at separate locations and process separate elements on the display for feedback information. This was achieved by independently changing the color of the tracking cursor every $5 \mathrm{sec}$ and the memory probe every $10 \mathrm{sec}$, according to the subject's momentary performance. The color represented the level of the subject's performance relative to a performance standard. The performance standard was determined individually for each subject for each task condition. Details on the algorithm for determining the standards are provided in the Procedure section below.

The color of the tracking cursor ranged from dark blue when RMSE was at least .02 lower (better) than the standard, light blue when RMSE was between .02 and .01 lower than the standard, white when RMSE was within .01 of the standard, yellow when RMSE was between .01 and .02 higher than the standard, and red if performance was at least .02 higher than the standard. The color 
of the memory probe ranged from dark blue when RT was at least $40 \mathrm{msec}$ faster than the standard, light blue when RT was between 25 and $40 \mathrm{msec}$ faster than the standard, white when RT was within $25 \mathrm{msec}$ of the standard, yellow when RT was between 25 and $40 \mathrm{msec}$ slower than the standard, and red when RT was at least $40 \mathrm{msec}$ slower than the standard.

\section{Design}

The subjects were randomly assigned to the three temporal predictability conditions. Half of the subjects in each predictability group performed the tracking task as the primary task; the other half performed the memory task as the primary task. There were 5 subjects in each of the six groups ( 3 predictability $\times 2$ priority groups).

Table 1 presents an overview of the experimental procedure. There were four sessions, each ranging from 2 to $2 \frac{1}{2} \mathrm{~h}$ in duration. The first three sessions served to stabilize single-task and equalpriority dual-task performance so that stabilized performance standards could be established for the secondary task trials. Single-task practice was intermixed with dual-task practice because single-task performance often improved after dual-task practice. On-line performance feedback was introduced at the end of Session 3. On-line feedback was not displayed earlier to avoid providing the subjects with unstable performance standards. However, it was important to familiarize the subjects with feedback prior to the secondary task technique trials so the feedback could be used effectively. The secondary task technique was introduced in the last session. In sum, the entire experiment consisted of four single-task blocks (S1-S4), three dual-task blocks (D1-D3), and two secondary task technique blocks (2T1 and 2T2). Further details can be found in Tsang and Shaner (1992).

\section{Procedure}

In each session, the subjects were asked to devote all their attention to the tasks and were encouraged to perform their best at all times throughout the experiment. At the end of each trial, the subjects were provided with verbal feedback on their performance (trial means). The subjects performed the easy conditions before the difficult conditions in Session 1. Task orders were randomized within each block in the remaining sessions. All subjects followed the same order in Sessions 1-3. A single-task block typically consisted of two trials of TR1 and SB2 and three trials of TR2 and SB4; a dual-task block consisted of two or three trials of each of the dual-task conditions.

On-line feedback was introduced in Session 3 and remained in effect until the end of the experiment. The feedback block contained one trial of each of the single-and dual-task conditions. The standards used in Session 3 for on-line feedback purposes were the subject's own best dual-task performance. The subjects were not given any performance standards explicitly; however, since they were to perform their best in both tasks (equal-priority dual tasks), they were asked to try to attain dark blue and to avoid red for both tasks.

The subjects performed two blocks of dual-task trials with the secondary task technique in Session 4 . The first block consisted of

Table 1

\begin{tabular}{cccc}
\multicolumn{4}{c}{ Procedure Overview } \\
\hline \multicolumn{3}{c}{ Session } \\
\hline demo & 2 & 3 & 4 \\
S1 & S2 & warm-up & warm-up \\
D1 & D2 & D3 & S4 1 \\
& S3 & FBS1 & $2 \mathrm{~T} 2$ \\
& & FBD2 & \\
\hline
\end{tabular}

Note- $\mathrm{S}=$ single-task block, $\mathrm{D}=$ dual-task block, FBS = single task with feedback, FBD = dual task with feedback, $2 \mathrm{~T}$ = secondary task technique. Numbers following these abbreviations refer to the block number. three trials and the second block consisted of two trials of each of the dual-task conditions. To minimize order effects, half of the subjects used Order A in the first block (2T1) and Order B in the second block (2T2). The other half of the subjects used Order B first.

At the beginning of the secondary task trials, the subjects were given a performance standard for the primary task. The primary task performance standard was determined by the subject's own best single-task performance (lowest tracking RMSE or the fastest RT associated with the highest accuracy achieved for the memory task). This was the standard used to drive the primary task color feedback. The secondary task standard used to drive the color feedback was the subject's best equal-priority dual-task performance. However, the subjects were not provided with any explicit performance standard for their secondary task performance. The subjects were instructed to perform their primary task performance as close as possible to the primary task standard, maintaining the feedback color white. However, they were instructed to perform their best with the secondary task and were told that the feedback color dark blue would indicate that they were doing well. It was emphasized to the subjects that maintaining primary task performance at the standard level was more important than performing the secondary task well.

A bonus system was used to provide additional incentive for the subjects to follow the priority instructions. The closer the primary task performance was to the primary task standard, the more points the subjects could earn. A smaller amount could also be earned by performing the secondary task well. This encouraged the subjects to place a high priority in maintaining the primary task standard without ignoring the secondary task entirely. The subject received a monetary bonus that ranged from $\$ 1$ to $\$ 5$ depending on the total number of points collected for the session. ${ }^{2}$

\section{RESULTS}

Since the present study aimed at discerning the mechanisms responsible for dual-task interference, it was deemed necessary to conduct the data analysis at a finer resolution than that of the trial means. Results of the trial means analysis have been presented in detail in Tsang and Shaner (1992) and are only summarized here to provide context for the moment-by-moment analysis.

\section{Summary of the Trial Means Analysis}

Six major conclusions were drawn from the trial means analysis. One, performance of the experimental tasks was resource-limited-a necessary condition for studying resource-related phenomena. This was supported by significant practice effects, significant difficulty effects, and significant dual-task decrements.

Two, no difference in baseline performance was observed among the six groups of subjects ( 3 predictability $\times 2$ priority). That is, none of the six groups had a predisposition to perform one task better than the other with the eventual priority manipulations.

Three, tracking error decreased and control speed increased with practice, whereas tracking error increased and control speed decreased with the addition of the memory task. This relationship between the tracking error and the tracking control speed suggested that the reduction in tracking error was effected by an increase in control speed.

Four, strong priority effects were observed. A withinsubject comparison between the differential-priority 
(primary or secondary task) and equal-priority dual-task performance was conducted on the primary tracking and primary memory groups separately. Primary task performance improved and the secondary task performance degraded relative to the equal-priority performance. The primary task control speed was higher than that of the equal-priority task in the second-order tracking conditions and in the high-memory-load conditions. This suggested that the subjects had to work harder to reduce the primary task error under the more difficult conditions. Furthermore, the secondary task control speed was higher than the equal-priority control speed when tracking was paired with the low-memory-load task but not with the high-memory-load task. This suggested that, in addition to protecting their primary memory task, the subjects also were working harder on the secondary tracking task but were able to do so only when the primary task was relatively easy.

Priority effects were also observed in a betweensubject comparison between the primary and secondary task performance. Primary task performance was superior to secondary task performance. It would be difficult to attribute this between-subject difference to the subjects' meeting demand characteristics.

Five, the difficulty of the two tasks had an asymmetric effect on each other's performance. Under the equalpriority condition, tracking order affected the memory RT but memory load did not affect tracking performance. However, under the differential-priority condition, the memory load affected both the memory and the tracking performances.

Six, no predictability effect was observed except that the performance operating characteristics (POCs) show that the VarI group did not adhere to the priority instructions as closely as did the two predictable groups. Between the two predictable groups, the FixR group appeared to time-share more efficiently than did the FixI group. The Fix I group (Figure 1b) appeared to have the most tradeoff, and the VarI group (Figure 1c) appeared to have the least. The box-like POC portrayed in Figure la suggests that the FixR group was the most efficient (i.e., closest to perfect time-sharing). There were two notable differences between the VarI group and the two predictable groups: (1) single-task memory RT from the VarI group was noticeably (though not statistically) slower than was that from the other two groups, and (2) the VarI group's RT improved in the direction dictated by priority but did not show the clear primary and secondary task RT differences, as did the two predictable groups (compare the horizontal distance between the Ts and the Ms across the three POCs). Between the two predictable groups, the FixR and FixI groups had comparable primary task performance, but the FixR secondary task was superior to that of the FixI.

The trial means analysis demonstrated many timesharing effects that are commonly observed in empirical tests of resource theories. However, outcome conflict can account for the dual-task performance decrement and the priority effects equally well. For example, superior primary task performance could be achieved by devoting more time to the task or by performing it first. The effectiveness of resource scarcity and outcome conflict in explaining the interference effects will be tested more closely by examining the moment-by-moment data.

\section{Moment-By-Moment Analysis}

The frame-by-frame tracking error and control speed from $5 \mathrm{sec}$ around the onset of the memory probe were ensembled over a trial. These were then ensembled over trials and subjects for each task condition. The $5 \mathrm{sec}$ started $1 \mathrm{sec}$ prior to the onset of each memory probe. There were a total of 15 tracking measures in $5 \mathrm{sec}$. Each of these measures was referred to as a window. The memory probe occurred in Window 4 . All post hoc comparisons conducted followed the Tukey procedure.

Equal-priority dual task. The moment-by-moment data from the equal-priority condition were examined first to provide a baseline for evaluating the priority effects. A block (D1-D3) $\times$ predictability $\times$ tracking order $X$ memory set size $X$ window (1-15) analysis of variance (ANOVA) was performed on the ensembled tracking error and control speed. A distinct, albeit transient, increase in tracking error was apparent slightly after the onset of the memory probe (Figure 2). Trend analysis indicated a significant quadratic trend in both the first-order $(p<.01)$ and the second-order $(p<.001)$ errors in each block.

To examine the transient error increase in a more analytical fashion, the following characteristics of the error increase were determined: the window in which the onset of the transient increase occurred, the window in which the peak occurred, the magnitude of the peak (highest error minus lowest error among the 15 windows), the recovery window in which the error returned to baseline, and the duration of the transient error increase (number of windows between onset and recovery). Onset was defined as the window with the smallest prepeak error that was not significantly different from the peak error. Recovery was defined as the window with the largest postpeak error that was not significantly different from the peak error. Post hoc comparisons, with $p<.05$, were used to determine the windows in which onset and recovery occurred.

Figure 2 shows that the first-order error peaked at Window 8 in Block D1 and peaked a little earlier at Window 7 in Blocks D2 and D3. The peak was significantly higher than all the windows before Window 7 in Block D1, Window 6 in Block D2, and Window 5 in Block D3. Error returned to baseline (recovery) in Window 10 in all blocks. Second-order error peaked later than did firstorder error at Window 10 in Blocks D1 and D2 and at Window 9 in Block D3. The onset of the error increase also occurred later for second-order than for first-order tracking. Onset occurred at Window 8 and recovered at around Window 13. Magnitude of the error peak was comparable for the two orders of control.

Figure 3 shows a transient tracking-error increase in each of the memory conditions. A significant quadratic 

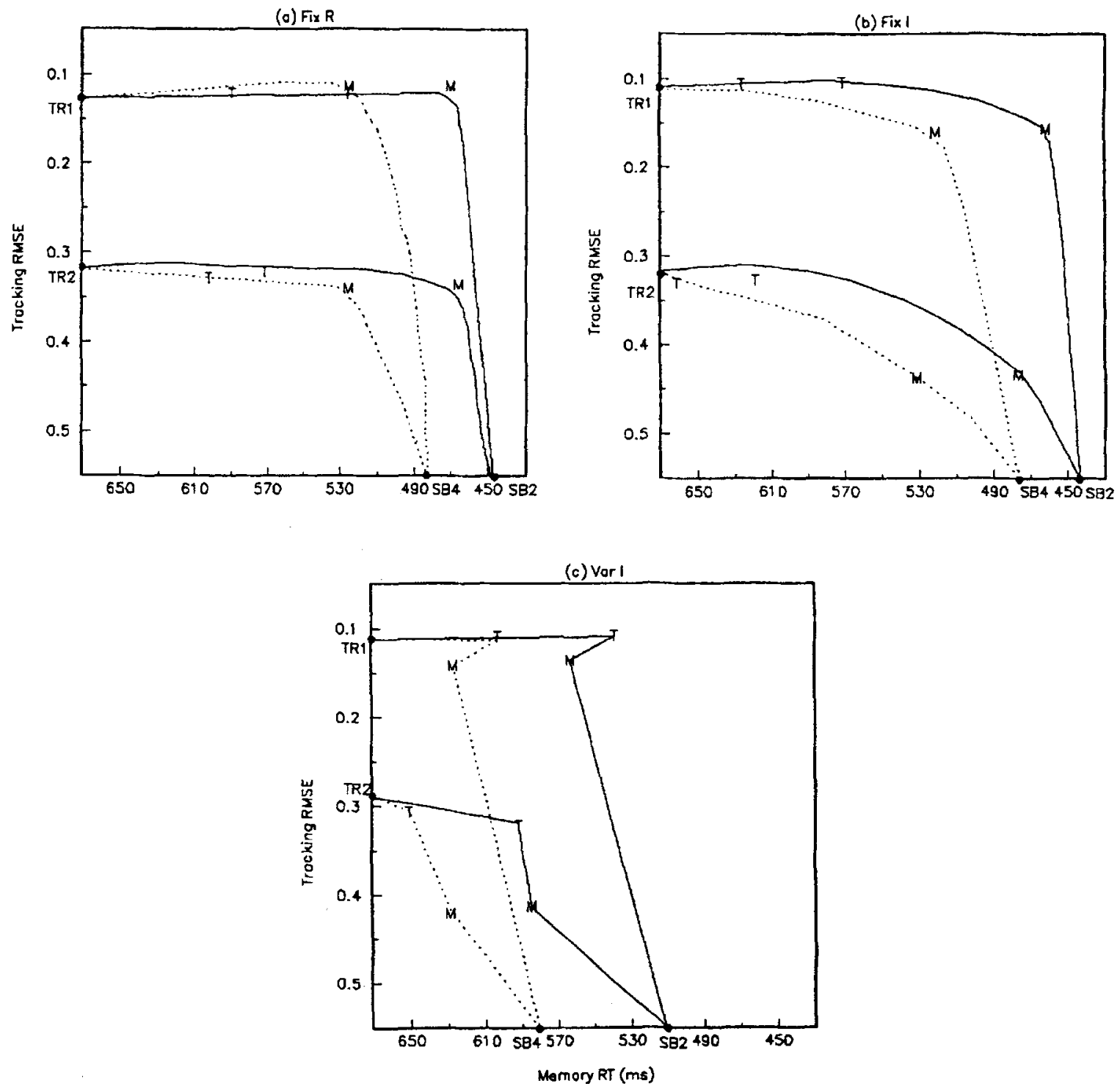

Figure 1. Performance operating characteristics (POCs) for the three predictability groups. Primary task standards (single-task performance) are plotted on the axes. The four dual-task conditions can be identified by the endpoints of each line on the axes. The top two lines in each POC are from the first-order tracking conditions, solid lines from the low-memory-load conditions. T denotes primary tracking, and $M$ denotes primary memory conditions. The lines are eyeball fits of the $T$ and $M$ datapoints.

trend $(p<.01)$ was observed in each block. Memory load had practically no effect on the tracking error.

Associated with the transient error increase was a momentary decrease in control speed (Figures 2 and 3). Substantiating the transient decrease in control speed were significant $(p<.05)$ quadratic trends detected in all four task pairs for the FixR and VarI groups. The only significant trend detected for the FixI group was a quartic trend $(p<.01)$ in TR1SB4.

All control speed troughs occurred at around Window 6, but first-order troughs were not significantly lower than the other windows. The magnitude of the control speed troughs was comparable for the two memory conditions (Figure 3), but second-order troughs were considerably larger than first-order troughs (Figure 2). This is interesting given that the magnitude of error increase was comparable for the two orders of control (Figure 2).

Since there was no priority distinction during the equal-priority condition, the two priority groups were collapsed in the analyses performed above. To check for random group differences, a predictability $\times$ priority (primary tracking vs. secondary tracking or primary memory groups) $\times$ tracking order $\times$ memory set size $\times$ window ANOVA was performed on the data from the last 

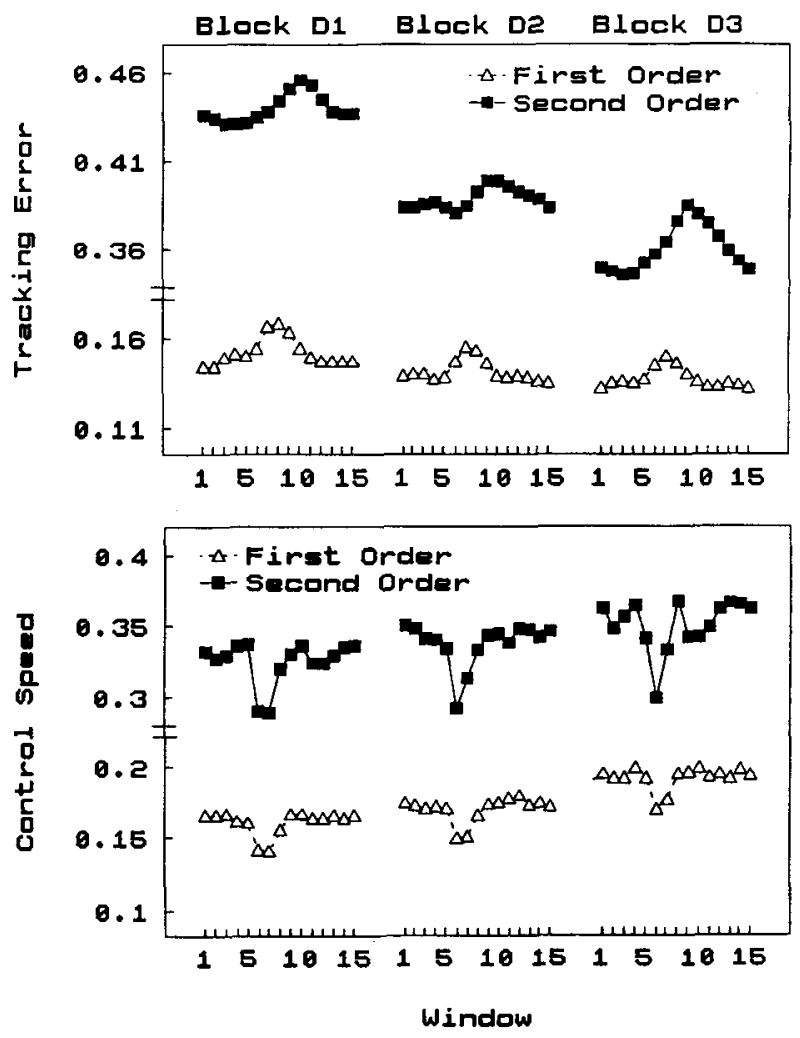

Figure 2. Ensembled averages of first- and second-order tracking error and control speed around the memory probe (Window 4) from the equal priority dual-task blocks.

equal-priority block (Block D3), where performance would be more stable. No significant predictability or priority effect was detected.

To summarize the findings obtained under the equalpriority condition, task interference was clearly manifested in the window effects. In response to the memory probe, tracking error increased momentarily, whereas control speed decreased momentarily. The error peaks for the two orders of control had similar magnitude, but second-order control had larger reduction in control speed. Memory load did not affect the magnitude of the error peaks or the control speed troughs. The onset of the control speed troughs did not change with practice, but practice sped up the recovery from the troughs. Finally, temporal predictability of the memory probes had little effect on the tracking response under the equalpriority condition, and no reliable difference between the priority groups was observed.

Differential-priority dual task. The two blocks of secondary task trials were collapsed and analyzed by predictability $\times$ priority (primary task vs. secondary task) $\times$ tracking order $\times$ memory set size $X$ window ANOVAs. The same characteristic transient increase in error and decrease in control speed were observed in the differentialpriority condition as in the equal-priority condition. Figure 4 shows that the secondary task error peaks and control speed troughs were larger than those of the primary task. In addition, the secondary task error level across the 15 windows was markedly higher than that of the primary task. Importantly, the higher secondary task error level was not accompanied by lower secondary task control speed level across the 15 windows, except for the larger trough. That is, the subjects did not appear to be ignoring the secondary task in general.

In contrast to the equal-priority condition, there were interesting priority and predictability interactions under the differential-priority condition. A priority $\times$ window interaction showed that the primary task tracking error peaked later (at Window 11) than did the secondary task tracking error (at Window 8) $[F(14,336)=3.40, p<$ .001 ; Greenhouse-Geisser $\left.(\mathrm{G}-\mathrm{G}),{ }^{3} p<.1\right]$. This suggested that the tracking error was sensitive to the memory response, since the slower secondary task memory response would delay its resource demand from, or interference with, the primary tracking task. The control speed troughs, on the other hand, did not move with the error peak and occurred at Window 6 regardless of priority. This indicated that the timing of the error peaks was not entirely dictated by the timing of the control speed troughs.

The tracking order $\times$ window interaction $[F(14,336)=$ $5.24, p<.001 ; \mathrm{G}-\mathrm{G}, p<.05]$ and a weak memory set size $\times$ window interaction $[F(14,336)=1.89, p<.05 ; \mathrm{G}-\mathrm{G}$,
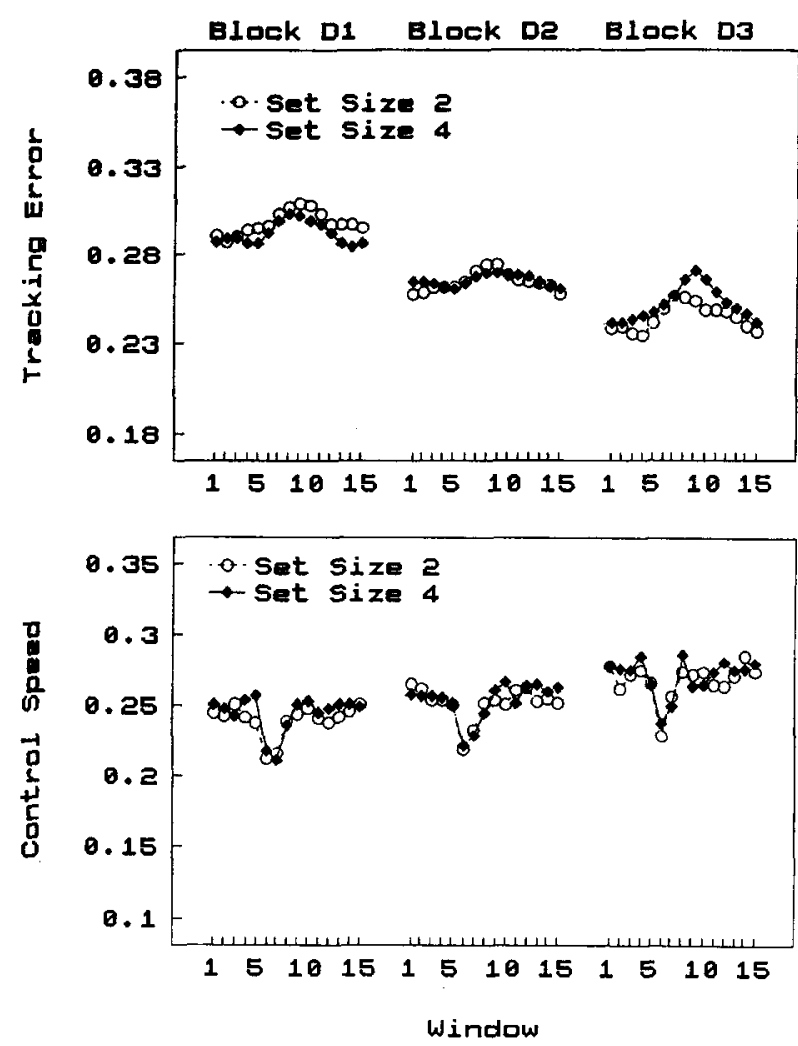

Figure 3. Ensembled averages of tracking error and control speed around the memory probe (Window 4) in high- and low-memoryload conditions from the equal-priority dual-task blocks. 
$p>.1]$ showed that the tracking error peaked later under the more difficult conditions. First-order error peaked at Window 7, low-memory-load error peaked at Window 7, high-memory-load error peaked at Window 8 , and secondorder error peaked at Window 10 . The later windows could be attributed to slower RT associated with the more difficult conditions, but there was no difference in the response demand between the high- and low-memory-load conditions. The memory-load effect thus suggested additional perceptual/central interference between the two tasks.

A weak three-way predictability $\times$ priority $\times$ window interaction in the tracking error $[F(28,336)=1.84, p<$ $.01 ; \mathrm{G}-\mathrm{G}, p>.1]$ is portrayed in Figure 5a. The FixR's primary task windows were relatively flat, and its secondary task error level was only slightly higher than that of the primary task. That is, the FixR group protected primary tracking performance at only a small cost to secondary tracking performance (see also Figure 1a). This interaction suggested that the FixR timing was advantageous to time-sharing.

As further support for the FixR advantage, the low primary task error from the FixR group was not associated with particularly high control speed (Figure $5 b$ ). That is, the FixR group did not appear to have to work particularly hard to achieve the superior tracking per-

(a)

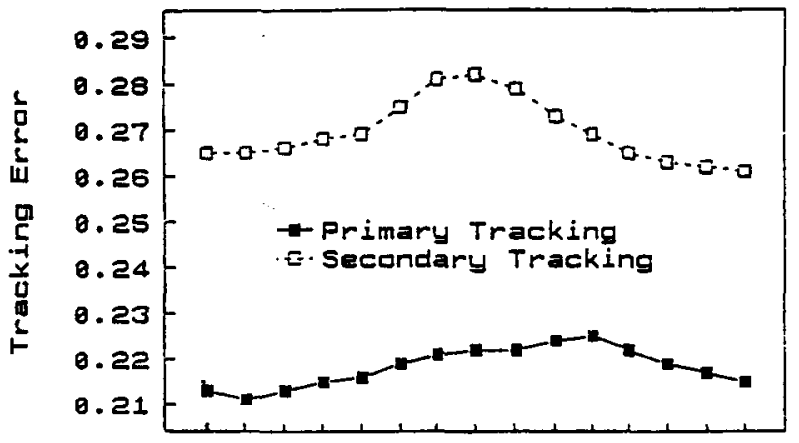

(b)

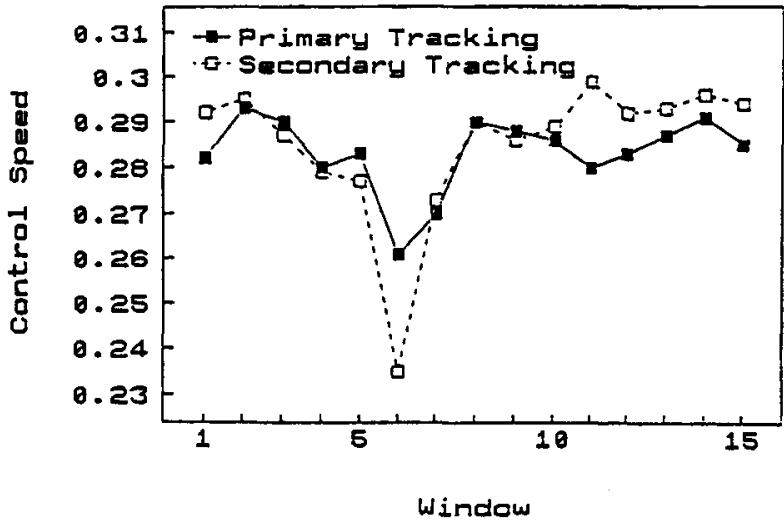

Figure 4. Ensembled averages of the primary and secondary tracking error and control speed around the memory probe (Window 4).

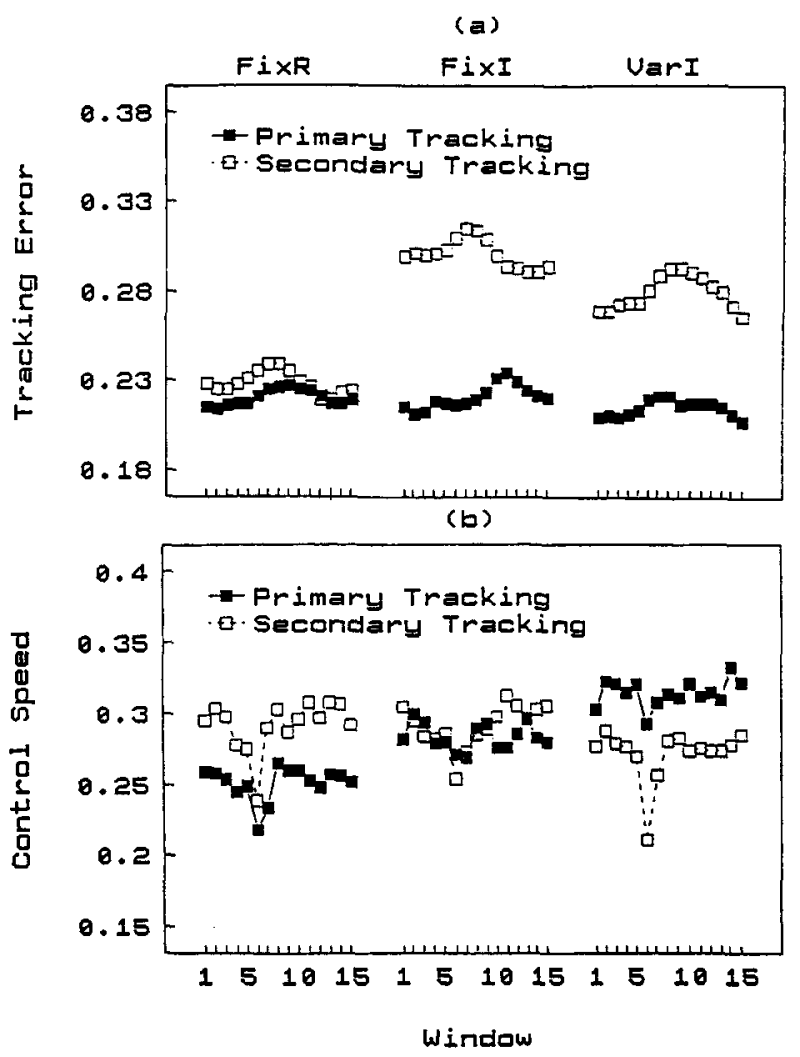

Figure 5. Predictability $\times$ priority $\times$ window interaction in tracking error and control speed.

formance. The superior primary tracking performance was also accompanied by relatively fast secondary task RT. Figure 5b shows that FixR's relatively low secondary task error was accomplished with considerable stick activity or high control speed. The Fix R subjects appeared then to have residual attention left for the secondary task. The pattern of results was quite different for the VarI group. The primary task error of the VarI group (Figure 5a) was achieved with considerable stick activity (Figure $5 b$ ).

To summarize, there were clear priority effects under the differential priority condition. Both the secondary task error peaks and control speed troughs were larger than those of the primary task, suggesting reduced stick activity for the low-priority task. Dissociations between the error and control speed measures suggested that the tracking error was not entirely determined by the control activity. First, the higher secondary task error level across the 15 windows was not associated with a lower overall control speed level (Figure 4). Second, the primary task error peak tended to occur later than the secondary task error peak, but the control speed trough remained at Window 6 regardless of priority (Figure 4). Also, the tracking-error peak was larger and occurred later with the more difficult conditions associated with slower RT. This suggested that the interference between the two tasks was related to response interference, but 
effects of memory load suggested additional central processing interference. Finally, the three-way interaction (Figure 5) suggested that the FixR group was best able to protect the primary task with little cost to the secondary task. But this interaction was not significant after the G-G correction.

\section{DISCUSSION}

The present research examined the efficacy of the resource-scarcity and outcome-conflict explanations of dual-task interference. A discrete-continuous task pair was purposely chosen to allow fine-grained analysis of time-shared performance. Outcome conflict could only be prevented by switching to avoid simultaneous processing. Graded-performance changes with the priority manipulation are intrinsic to, and would be supportive of, resource theories.

\section{Dual-Task Interference}

Transient tracking-error increase accompanied by transient drop in control speed was observed around the time that the response to the memory probe occurred. This form of interference resembled that observed in a number of the studies reviewed earlier. On the surface, this interference pattern is consistent with the resourcescarcity and outcome-conflict explanations. However, deeper analysis revealed that outcome conflict cannot easily explain several aspects of the data.

Temporal relation between the discrete and the continuous response. The present results and the results of many of the studies reviewed show that the interference was temporally tied to the concurrent discrete response, as opposed to the onset of the stimulus. This suggests that the interference occurred primarily during response processing as opposed to the perceptual processing of the stimulus. Because the interference in tracking response was observed to begin prior to the discrete RT, Klapp et al. (1987) and Netick and Klapp (1994) argued that the interference arose during the preparation or programming of the response as opposed to during response execution. The interference was attributed to a singlechannel processor that is constrained to programming one response at one time.

The present data indicate that the interference did not arise only during response preparation. Figure 6 displays the temporal relations between the response to the memory probe and the tracking activity in the equal-priority conditions. Only data from Block D3 are displayed to focus on the more stable performance. The memory probe was presented during Window 4 , and each window was $333 \mathrm{msec}$ in duration. The discrete RTs tended to occur at the end of Window 5 or the beginning of Window 6 across the four dual-task conditions. The onset of the control speed decrease occurred in Window 5 or between 333 and $667 \mathrm{msec}$ after the onset of the memory probe (see Figures 2 and 3). This temporal overlap between the discrete response and the start of the reduction in tracking control is consistent with Netick and Klapp's contention of response programming interference. However, the maximum interference did not occur until later. The peak of the first- and second-order transient error increase occurred in Windows 7 and 9, respectively. In other words, first-order tracking error did not reach its peak until at least one third of a second after the discrete response, and the second-order error did not reach its
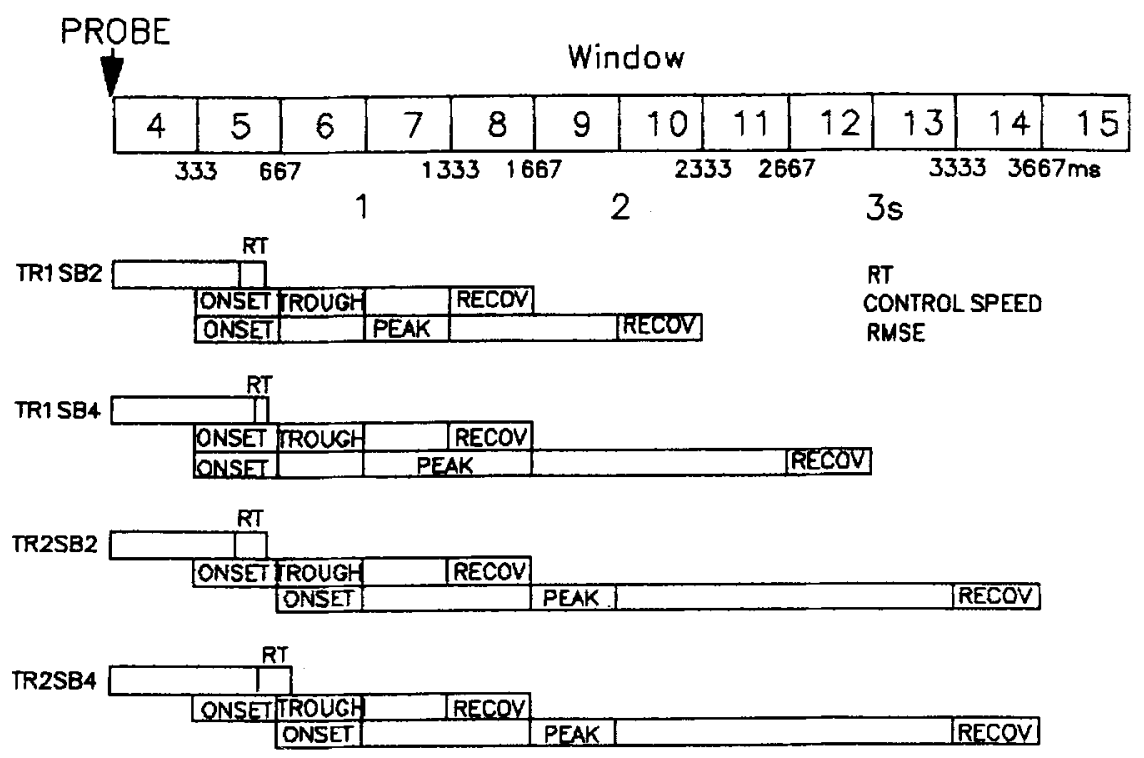

Figure 6. Temporal relation between the discrete memory task response and the continuous tracking response for each dual-task condition obtained from Block $\mathrm{D3}$ under the equal-priority condition. Each window was 333 msec in duration. The range of reaction times displayed is the range of the three predictability groups. $\mathrm{RECOV}=$ recovery to baseline performance. 
peak until $1 \mathrm{sec}$ or more had elapsed. The fact that it might take some time for error to accumulate could account for the relatively late error peaks. But the maximum decrease in control speed (Window 6), which is a more direct measure of the tracking activity than is RMSE, also occurred after the discrete RT. Recovery of control speed did not occur until Window 8-between two thirds of a second to one full second after the discrete RT. Similar results were reported in Vidulich (1989, 1990). Interference between the two responses thus went beyond that of response preparation.

That the control speed troughs occurred beyond the discrete RT showed that dual-task interference was not confined to response interference. Even after the discrete response, there were potential conflicts of resource demands. The subjects still had to continuously maintain the memory set in working memory while simultaneously processing the tracking task. It is possible that recovering from a disruption to tracking is momentarily more demanding than is maintaining a steady-state tracking performance. If a fairly constant amount of resources or processing was needed to maintain the memory set, there would have been competition for resources or processes between maintaining the memory set and recovery from disruption to the tracking task. This competition would have been especially damaging to the second-order tracking because of its strong perceptual/ central processing requirements. This central interference is further supported by evidence presented below.

Dissociation between the tracking error and control speed. Since control speed is a more direct measure of the subject's motor response, response competition or outcome conflict would be expected to manifest in the control speed measure. Dissociation between tracking error and control speed suggested that tracking error was not determined solely by motor activity. Five major dissociations were of note. One, although second-order control tracking had larger control speed troughs than did first-order control, second-order control did not produce significantly higher error peaks (Figure 2). This suggests that control speed was reduced by reduced deflection of the stick as opposed to releasing the stick and allowing it to return to center or by holding the stick still (freezing; see Klapp et al., 1987; Netick \& Klapp, 1994). The reason is that the latter two mechanisms should lead to differential error magnitude for the two orders of control.

First, returning the stick to center would lead to zero cursor velocity in first-order control and would therefore maintain the magnitude of error rather than propagate the error. In contrast, error would continue to accumulate with second-order control because, although the cursor acceleration would be zero, cursor velocity would not be. Second-order error should therefore continue to accumulate after the stick has been returned to center. Firstand second-order controls should thus have different error peak magnitudes when the stick is returned to center. This was not observed. Netick and Klapp (1994) also did not find support for this centering strategy.
Second, holding the stick still would maintain the current velocity of the cursor with first-order control, so error would continue to accumulate. Holding the stick still with second-order control would maintain the current acceleration of the cursor, and error should accumulate even faster than with first-order control. Larger, if not earlier, second-order error peak would be expected with the holding strategy. This was not observed (Figures 2 and 6).

If subjects were not holding the stick still or releasing the stick, they had to have been moving the stick. The momentary decrease in control speed indicated that the subjects simply reduced the extent of stick deflection, leading to reduced control speed. Note that the same amount of reduction in the extent of the stick deflection would lead to greater speed loss in second-order control than in first-order control. This would explain the larger second-order control speed trough. Levison, Elkind, and Ward (1971) also observed a decrease in the size of control movements as processing demand increased. These researchers proposed that the decrease was probably to reduce the chance that an incorrect movement would cause the subjects to lose control of the system.

In sum, the dissociation between the control speed troughs and error peaks indicate that the subjects were not releasing control of the stick or freezing at the time of the memory response. Although tracking activity was reduced at the time of the memory response, the tracking error in Block D3, even at its peak, was not as high as the baseline error obtained early in practice in Block D1 (Figure 2). This and the dissociation strongly suggest that, although there was a reduction in tracking activity, the subjects did not abandon tracking control completely at the time of the memory response. In short, the subjects did not appear to be adopting a switching strategy.

The other four dissociations suggest central interference in addition to response interference. Two, although the control speed for both orders of control recovered in Window 8 under the equal-priority condition, secondorder error persisted longer (slower recovery) than did first-order error (Figure 6). Since the tracking error peak was about the same for both orders of control, secondorder control did not have higher error to recover from. Three, increased secondary task error level was not accompanied by lower secondary task control speed level (Figure 4). Four, although the error peaks occurred earlier with practice (Figure 2) and were delayed with higher priority (Figure 4), the control speed troughs remained at Window 6 (Figures 2 and 4). Five, under the differential-priority condition, the tracking error peaked at Window 7 with the low-memory-load condition and at Window 8 with the high-memory-load condition, without the corresponding changes in control speed. Note that the response demand did not change with memory set size; response selection, preparation, and execution for the discrete response should be the same.

The dissociations showed that the interference was not entirely motor in nature (see also Cliff, 1973; Klapp 
et al., 1987; McLeod, 1977; Netick \& Klapp, 1994; and Vidulich, 1989, 1990). That the interference in the tracking activity lasted considerably beyond the discrete RT suggests that the interference cannot be attributed entirely to the response programming or even response execution of the two tasks (see also Vidulich, 1989, 1990). The memory-load effect further supports the notion that central interference was also involved.

\section{Causes of Interference}

According to resource theories, resource scarcity necessitates graded-performance tradeoffs in response to priority changes, and predictability of stimulus arrival should facilitate resource allocation control. To avoid outcome conflict, simultaneity of the competing tasks or processes has to be avoided. Priority and predictability may alter the switching schedule but graded-performance changes at the moment-by-moment level would not be consistent with the outcome-conflict explanation. The interference patterns will now be evaluated in light of these predictions.

Priority effects. Performance tradeoff obtained with priority instructions is accepted as an essential demonstration of resource allocation between time-shared tasks even by critics of the resource view (Navon, 1984). Clear priority effects were observed in both the trial means and the moment-by-moment data. Primary task had faster RT, lower tracking RMSE, smaller momentary tracking error peaks, and smaller control speed troughs than did the equal-priority and secondary tasks. The primary tracking task also had higher control speed level than did the equal-priority task. This suggests that the subjects, in general, worked harder in order to achieve the lower primary task tracking error. In conjunction, secondary task had slower RT, higher tracking RMSE, and larger control speed troughs than did the equal-priority and primary tasks.

Priority effects were also observed in a number of studies reviewed earlier. Klapp et al. (1987) found the frequency of tracking holds and holds of long duration to decrease with increased tracking priority. Tsang and Wickens (1988) found that subjects were better able to protect the primary task in the manual-manual task pairs than in the manual-speech task pairs from error spikes, despite a greater level of interference (larger dual-task performance decrement) in the manual-manual task pairs. Vidulich (1990) found that the high-priority, but not the low-priority, memory task response intruded on the tracking performance (larger error peaks).

One main criticism of previously observed performance tradeoffs has been that certain priority manipulations could implicitly convey to the subjects that the task performance should tradeoff (Navon, 1984). The observed tradeoff may thus mean nothing more than that the subjects are complying with demand characteristics. But the priority effects observed in the present experiment could not be attributed easily to the subjects' complying with tradeoff expectations, given the present priority in- structions and the between-subject priority manipulation. The present priority effect could also not be attributed to a simple between-subject difference, since no group main effect was detected in the single- and equal-priority dualtask performance. Performance was progressively worse from the high-priority condition (primary task), equalpriority condition, to the low-priority condition (secondary task). How interference could occur less frequently (e.g., Klapp et al., 1987) and how task priority (an instructional variable) could affect the extent of the interference (e.g., Tsang \& Wickens, 1988) cannot be accommodated easily within the outcome-conflict view, given that the dual-task interference described above does not support a switching strategy. The performance tradeoff observed, therefore, provides strong support for the resource view.

Predictability effects. Voluntary control of attention allocation was implicated by the predictability $\times$ priority $X$ window interaction. This interaction suggests that the performance tradeoffs were not mere results of the subjects' meeting demand characteristic, since all three predictability groups received the same priority instructions. Figure 5a shows that the FixR group not only performed at an overall lower error level but also had smaller error peaks. As proposed earlier, the self-generated timemarker in the FixR condition could perhaps be used more effectively to anticipate the next memory probe, enabling the FixR group to better monitor the level of attention required to protect the tracking task. This would be highly consistent with the graded-performance changes among the primary task, equal-priority, and secondary task conditions.

The outcome-conflict view could also explain a predictability main effect. For example, with predictable stimulus arrival, subjects could schedule the different processes so as to minimize outcome conflict. However, the outcome-conflict view could not easily explain the interaction between the predictability and priority variables. No predictability main effect was observed in the equal-priority condition. That the subjects would only try to avoid outcome conflict under the differential priority condition does not seem plausible, since both the instructions and the on-line feedback encouraged the subjects to perform their best throughout the experiment. There was also no evidence of response shuffling in response to priority changes. The tracking-error peak tended to be temporally tied to the discrete response regardless of priority. Figure 4 shows that the secondary task tracking-error peak occurred earlier than did that of the primary task. Note that the primary memory RT (concurrent with the secondary tracking response) was faster than the secondary memory RT.

A caveat in interpreting the predictability interaction is that it was not significant after the Greenhouse-Geisser correction. Even though the correction tends to overcorrect, the small $F$ value indicates that the predictability effect was, at best, weak. The distinction between the resource-scarcity and outcome-conflict explanations based on the present predictability effect is therefore only ten- 
tative at this point. Figures 1 and 5 suggest that the effects of predictability should be examined more closely in future research.

Asymmetric interference. In the equal-priority condition, the difficulty of the two tasks was found to have asymmetric effects on each other's performance: tracking order had robust effects on the performance of both tasks, but memory load did not affect tracking performance. Under the differential-priority condition, the trial means analysis shows that memory load affected the primary task RMSE and the primary and secondary task control speed. The moment-by-moment analysis revealed that the primary task tracking error peaked later and the secondary tracking task had larger control speed troughs under the high-memory-load condition. These results can be interpreted easily within a multiple-resource framework.

Within a multiple-resource framework (Wickens, 1990), the memory task required mostly perceptual/central processing resources for maintaining and scanning the memory set. Diverting central processing resources away from the memory task should therefore have deleterious effect on performance. The deleterious effect would be magnified with second-order tracking due to its central processing demand from the need to anticipate acceleration effects-hence, the order effect on memory performance. On the other hand, the tracking task relied heavily on the response-processing resources, and second-order tracking exerted an increased response load due to its higher response speed and its more complex response requirement. But the response-processing demand of the memory task was minimal (a buttonpress) and did not change with the memory load. Thus, while adding a memory task to the tracking task led to trackingperformance decrements, increasing the memory load did not further degrade the tracking performance in the equal-priority conditions. But, as additional resources were needed to meet increased priority demand and to manage resource allocation to comply with the specified priority, the high-memory-load task would have competed more for the central processing resources from the tracking task. Hence, the memory-load effect was evident only under the more intensive resource competition inflicted by the differential-priority condition.

Although the outcome-conflict view, on the surface, could explain the asymmetric interference by proposing that the more complex second-order task generated greater outcome conflict that affected both task performances, it does not offer any elegant explanation for the memoryload effect that was manifested only under the differentialpriority condition when there was no evidence of switching. By contrast, the resource view can readily account for both the asymmetric interference and the memoryload effect.

Finally, that the window in which the error peak occurred shifted with the RT as it improved with practice or as priority changed supports the notion that the observed interference had a large response interference component. Indeed, all the studies reviewed here sug- gest a response-related interference. However, this does not necessarily constitute support for the single-responsechannel view, given that common among the studies is a tracking task that is clearly a response-demanding task and all the discrete tasks involved relatively simple perceptual stimuli. Together with the memory-load effect, the interference observed in the present study is incongruous with the single-response-channel interpretation.

\section{Conclusion}

The present research demonstrated the utility of microanalysis of human performance. The present research also demonstrated that performance tradeoff could be obtained with an unbiased secondary task technique. Under properly controlled conditions, performance tradeoff is an important demonstration of resource scarcity. Also, the transient error increase and transient control speed decrease right around the response to the memory task certainly could be characterized as outcome conflict. But these results were also consistent with the resource explanation. In addition, the priority effect and possibly a weak predictability effect suggested a voluntary control that produced graded-performance changes. This can be easily accounted for by resource scarcity but difficult to interpret within the outcome-conflict view. However, the distinction between the resource-scarcity and outcome-conflict explanations based on the statistically weak predictability effect should be further tested with more powerful manipulations. The multiple-resource model offered an elegant account for the asymmetric interference that cannot be easily accommodated by a single-response-channel model. It is proposed in the present paper that outcome conflict could only be avoided by avoiding simultaneity of processing or by switching between the time-shared tasks. Failing to find support for the switching strategy, the resource view offers a more comprehensive and parsimonious explanation, even though several aspects of the dual-task interference would be consistent with the outcome-conflict explanation. However, the possibility of not having sufficiently high resolution or not having a sufficiently sensitive test for examining switching remains.

\section{REFERENCES}

BEATTY, J. (1982). Task-evoked pupillary responses, processing load, and the structure of processing resources. Psychological Bulletin, 91, 276-292.

BROADBENT, D. (1987). Structure and strategies: Where are we now? Psychological Research, 49, 73-79.

CLIFF, R.C. (1973). Attention sharing in the performance of a dynamic dual task. IEEE Transactions on Systems, Man, \& Cybernetics, SMC-3, 241-248.

FRACKER, M. L., \& WiCkENS, C. D. (1989). Resources, confusions, and compatibility in dual-axis tracking: Displays, controls, and dynamics. Journal of Experimental Psychology: Human Perception \& Performance, 15, 80-96.

Friedman, A., Polson, M. C., \& Dafoe, C. G. (1988). Dividing attention between the hands and the head: Performance trade-offs between rapid finger tapping and verbal memory. Journal of Experimental Psychology: Human Perception \& Performance, 7, 1031-1058. 
GOPHER, D. (1986). In defence of resources: On structures, energies, pools and the allocation of attention. In G. R. J. Hockey, A. W. K. Gaillard, \& M. G. H. Coles (Eds.), Energetics and human information processing (pp. 353-372). Boston: Martinus Nijhoff.

GOPHER, D. (1993). The skill of attention control: Acquisition and execution of attention strategies. In D. Myers \& S. Kornblum (Eds.), Attention and performance XIV (pp. 299-322). Hillsdale, NJ: Erlbaum.

GOPHER, D., BRICKNER, M., \& NAVON, D. (1982). Different difficulty manipulations interact differently with task emphasis: Evidence for multiple resources. Journal of Experimental Psychology: Human Perception \& Performance, 8, 146-157.

GOPHER, D., \& NAVON, D. (1980). How is performance limited? Testing the notion of central capacity. Acta Psychologica, 46, 161-180.

Hirst, W., \& KALMAR, D. (1987). Characterizing attentional resources. Journal of Experimental Psychology: General, 116, 68-81.

JAGACINSKI, R. J. (1977). A qualitative look at feedback control theory as a style of describing behavior. Human Factors, 19, 331-347.

Kahneman, D. (1973). Attention and effort. Englewood Cliffs, NJ: Prentice-Hall.

KEPPEL, G. (1991). Design and analysis: A researcher's handbook (3rd ed.). Englewood Cliffs, NJ: Prentice-Hall.

Kinchla, R. A. (1980). The measurement of attention. In R. S. Nickerson (Ed.), Attention and performance VIII (pp. 213-238). Hillsdale, NJ: Erlbaum.

Kinsbourne, M., \& Hicks, R. E. (1978). Functional cerebral space: A model for overflow transfer and interference effects in human performance. In J. Requin (Ed.), Attention and performance VII (pp. 345362). Hillsdale, NJ: Erlbaum.

KlapP, S. T,, Kelly, P. A., \& NeTick, A. (1987). Hesitations in continuous tracking induced by a concurrent discrete task. Human Factors, 29, 327-338.

LEVISON, W. H., ElKIND, J. I., \& WARD, J. L. (1971). Studies of multivariable manual control systems: A model of task interference (Report No. NASA CR-1746). Washington, DC: V. H. Winston.

MCLEOD, P. (1977). A dual task response modality effect: Support for multiprocessor models of attention. Quarterly Journal of Experimental Psychology, 29, 651-667.

MoRAy, N. (1967). Where is capacity limited? A survey and a model. Acta Psychologica, 27, 84-92.

MORAY, N. (1978). The strategic control of information processing. In G. Underwood (Ed.), Strategies of information processing (pp. 301327). New York: Academic Press.

NAvon, D. (1984). Resources: A theoretical soup stone? Psychological Review, 91, 216-234.

NAvon, D. (1985a). Attention division or attention sharing? In M. I. Posner \& O. S. M. Marin (Eds.), Attention and performance XI (pp. 133-146). Hillsdale, NJ: Erlbaum.

NAvon, D. (1985b). Do people allocate limited resources among concurrent activities? (IPDM Report No. 26). Haifa, Israel: Laboratory for Information Processing and Decision Making.

NETICK, A., \& KLAPP, S. T. (1994). Hesitations in manual tracking: A single-channel limit in response programming. Journal of Experimental Psychology: Human Perception \& Performance, 20, 766-782.

O'DonNell, R. D., \& EGgEMEIER, F. T. (1986). Workload assessment methodology. In K. R. Boff, L. Kaufman, \& J. P. Thomas (Eds.), Handbook of perception and human performance (Vol. 2, pp. 42/142/49). New York: Wiley.

OGden, G. D., LEVINE, J. M., \& EISNER, E. J. (1979). Measurement of workload by secondary tasks. Human Factors, 21, 529-548.

ONstotT, E. D. (1976). Task interference in multi-task aircraft stabi- lization. In Proceedings of the Twelfth Annual Conference on Manual Control (NASA TMX-73, 170, pp. 80-103). Moffett Field, CA: Ames Research Center.

ROLFE, J. M. (1971). The secondary task as a measure of mental load. In W. T. Singleton, J. G. Fox, \& D. Whitfield (Eds.), Measurement of man at work (pp. 135-148). London: Taylor \& Francis.

Sperling, G., \& Melchner, M. J. (1978). The attention operating characteristic: Examples from visual search. Science, 202, 315-318

STERNBERG, S. (1969). Memory scanning: Mental processes revealed by reaction time experiments. American Scientist, 57, 421-457.

TSANG, P. S., \& SHANER, T. S. (1992). Resource scarcity and intrusion in time-sharing performance (Tech. Rep. EPL-92-1). Dayton, $\mathrm{OH}$ : Wright State University, Engineering Psychology Laboratory.

Tsang, P. S., \& Wickens, C. D. (1988). The structural constraints and strategic control of resource allocation. Human Performance, 1 , 45-72.

VIDULICH, M. A. (1989, May). Resources and time-sharing performance: Testing the viability of resource theory with Navon's optimum maximum technique. Paper presented at the Fifth Mid-Central Ergonomics/Human Factors Conference, Dayton, $\mathrm{OH}$.

VIDULICH, M. A. (1990). Performance-based workload assessment: Allocation strategy and added task sensitivity. In Third Annual Workshop on Space Operations Automation and Robotics (SOAR '89) (NASA Conference Publication 3059, pp. 329-335). Washington, DC: National Aeronautics and Space Administration.

WELFORD, A. T. (1978). Mental work-load as a function of demand, capacity, strategy, and skill. Ergonomics, 21, 151-167.

WiCkENS, C. D. (1986a). The effects of control dynamics on performance. In K. Boff, L. Kaufman, \& J. Thomas (Eds.), Handbook of perception and performance (Vol. 2, pp. 39/1-39/60). New York: Wiley.

WICKENS, C. D. (1986b). Gain and energetics in information processing. In G. R. J. Hockey, A. W. K. Gaillard, \& M. G. H. Coles (Eds.), Energetics and human information processing (pp. 373-380). Boston: Martinus Nijhoff.

WiCKENS, C. D. (1990). Processing resources and attention. In D. Damos (Ed.), Multiple task performance (pp. 3-34). London: Taylor \& Francis.

Wickens, C. D., Gill, R., Kramer, A., Ross, W., \& Donchin, E. (1981). The processing demands of higher order of manual control. In J. Lyman \& A. Bejczy (Eds.), Proceedings of the 17th Annual Conference on Manual Control (pp. 81-95). La Canada, CA: Jet Propulsion Lab.

WICKENS, C. D., \& GoPHER, D. (1977). Control theory measures of tracking as indices of attention strategies. Human Factors, 19, 349-365.

\section{NOTES}

1. Jagacinski (1977) and Wickens (1986a) provide excellent overviews of the parameters and demand of manual control.

2 . The task and priority instructions in their entirety and a more detailed description of the bonus system can be found in Tsang and Shaner (1992).

3. Greenhouse-Geisser (G-G) corrected $p$ levels are reported along with the uncorrected levels because the G-G correction tends to overcorrect (Keppel, 1991).

(Manuscript received December 3, 1993; revision accepted for publication October 18, 1994.) 